\title{
Diagnosis and Clinical Management of Long-chain Fatty-acid Oxidation Disorders: A Review
}

\author{
Joshua J Baker ${ }^{1,2}$ and Barbara K Burton ${ }^{1,2}$ \\ 1. Ann \& Robert H. Lurie Children's Hospital of Chicago, Chicago, IL, USA; 2. Northwestern University Feinberg School of Medicine, \\ Chicago, IL, USA
}

DOI: https://doi.org/10.17925/EE.2021.17.2.108

L ong-chain fatty-acid oxidation disorders (LC-FAODS) are autosomal recessive inherited metabolic conditions that occur due to a disruption in the body's ability to perform mitochondrial beta oxidation. Expanded newborn screening is widening phenotypic understanding of these disorders, as well improving our knowledge of disease incidence. Management of these disorders is focused on avoidance of fasting, dietary changes and supplementation with energy sources that bypass the metabolic block. Recent US Food and Drug Administration approval of triheptanoin has improved the outcome for affected individuals. New research into dietary modifications and novel pharmacologic therapies continues for these disorders. In this article, we review the major LC-FAODS and their clinical presentation.

\section{Keywords}

Long-chain fatty-acid oxidation disorders (LC-FAODS), beta oxidation, triheptanoin, newborn screening, nutrition, inborn errors of metabolism

Disclosures: Barbara K Burton has received consulting fees and/or honoraria from Ultragenyx Pharmaceutical Inc. Joshua J Baker has no financial or non-financial relationships or activities to declare in relation to this article.

Review process: Double-blind peer review.

Compliance with ethics: This study involves a review of the literature and did not involve any studies with human or animal subjects performed by any of the authors.

Authorship: The named authors meet the Internationa Committee of Medical Journal Editors (ICMJE) criteria for authorship of this manuscript, take responsibility for the integrity of the work as a whole, and have given final approval for the version to be published.

Access: This article is freely accessible at touchENDOCRINOLOGY.com ( ) Touch Medical Media 2021 Received: 8 March 2021

Accepted: 13 April 2021

Published online: 10 September 2021

Citation: touchREVIEWS in Endocrinology. 2021; 17(2):108-11

Corresponding author: Joshua J Baker, Ann \& Robert H Lurie Children's Hospital of Chicago, Northwestern University Feinberg School of Medicine, 225 E Chicago Avenue, Chicago, IL, 60661, USA.

E: jobaker@luriechildrens.org

Support: No funding was received for the publication of this article.
Long-chain fatty-acid oxidation disorders (LC-FAODS) are pan-ethnic, autosomal recessive, inherited metabolic conditions causing disruption in the processing or transportation of fats into the mitochondria to perform beta oxidation. ${ }^{1}$ In normal metabolism, long-chain fatty acids are bound to carnitine within the cytosol of cells, and transported across the mitochondrial membranes Within the mitochondria, they are then cleaved from carnitine to undergo beta oxidation by a complex of enzymes specific to the length of the carbon chain. These enzymes go through cycles of beta oxidation until the carbon chain has been reduced to a structure that may feed into the tricarboxylic acid cycle for energy production. This energy source is critical when the body is in a fasting state, providing energy for skeletal and cardiac muscle, the liver and other tissues. ${ }^{2}$

Disorders result from defects in the transportation or utilization of these long-chain fats for energy, which become critical when the body has exhausted its glycogen stores. ${ }^{3}$ Classical symptoms shared among all disorders include hypoketotic hypoglycaemia, hepatic dysfunction, cardiomyopathy, skeletal myopathy, rhabdomyolysis and sudden death. ${ }^{1}$ However, there is wide variability within phenotypes that may range from critical illness in an infant, to exercise intolerance in an adult. Understanding of these presentations is increasing due to expansion in testing for these conditions, and a broader understanding of genotype-phenotype correlations. Early recognition of the diagnosis of LC-FAOD is important in optimizing outcomes. Even if patients with LC-FAODs are asymptomatic at presentation, most can be expected to suffer from recurrent decompensation during times of acute illness or stress, requiring acute and chronic medical management. ${ }^{4}$ In this narrative review we explore the current clinical approach to the diagnosis and management of LC-FAODS. A subjective literature review was conducted using PubMed, in addition to utilization of current clinical guidelines, with the aim of providing a concise resource for practicing clinicians and other healthcare professionals treating patients with LC-FAODs.

\section{Long-chain fatty-acid oxidation disorders}

Systemic primary carnitine deficiency

Systemic primary carnitine deficiency (CDSP), also known as carnitine transport disorder or carnitine uptake disorder, is caused by biallelic variants in SLC22A5. ${ }^{5}$ This disorder results in a defect in the transportation of carnitine from the serum into cells. Carnitine is essential in the transportation of long-chain fats from the cytosol into the mitochondria for beta oxidation. CDSP has a wide range of clinical prestations. The classic phenotype includes recurrent episodes of hypoketotic hypoglycaemia, elevated transaminases, hepatomegaly and hypoglycaemia. There is a severe childhood myopathic form that affects both skeletal and cardiac muscle, with increased risk for sudden death. Due to the implementation of newborn screening, mild forms have become more commonly diagnosed, with easy fatiguability or no clinical symptoms reported. ${ }^{6}$ Since carnitine is transported from maternal serum across the placenta during pregnancy, there have been many cases in which low carnitine on newborn screening is actually due to an underlying diagnosis 
Table 1: Classical biochemical findings for long-chain fatty-acid oxidation disorders

\begin{tabular}{|l|l|l|}
\hline Disorder & Acylcarnitine abnormalities & \\
\hline CDSP & $\downarrow \mathrm{CO}$ & $\uparrow$ Carnitine, nonspecific dicarboxylic aciduria \\
\hline CPT1A & $\uparrow \mathrm{C} 0, \mathrm{C} 0 / \mathrm{C} 16+\mathrm{C} 18 ; \downarrow \mathrm{C} 2$ & $\uparrow$ Dodecanedioic acid, $\mathrm{C} 12$ dicarboxylic acid \\
\hline CACT & $\uparrow \mathrm{C} 16, \mathrm{C} 18, \mathrm{C} 18: 1, \mathrm{C} 18: 2$ & \\
\hline CPT2 & $\uparrow \mathrm{C} 16, \mathrm{C} 18, \mathrm{C} 18: 1, \mathrm{C} 18: 2$ & \\
\hline VLCAD & $\uparrow \mathrm{C} 12, \mathrm{C} 14, \mathrm{C} 14: 1, \mathrm{C} 16, \mathrm{C} 18$ & $\uparrow$ Longer-chain dicarboxylic aciduria \\
\hline LCHAD & $\uparrow \mathrm{C} 14 \mathrm{OH}, \mathrm{C} 16, \mathrm{C} 16 \mathrm{OH}, \mathrm{C} 18 \mathrm{OH}, \mathrm{C} 18: 1 \mathrm{OH}$ & $\uparrow$ Longer-chain 3-hydroxydicarboxylic acids \\
\hline
\end{tabular}

$\mathrm{CO}=$ low levels of free carnitine; $C A C T=$ carnitine acylcarnitine translocase; $C D S P=$ systemic primary carnitine deficiency; $C P T 1 A=$ carnitine palmitoyltransferase $1 \mathrm{~A} ;$

CPT2 = carnitine palmitoyltransferase 2; LCHAD = long-chain 3-hydroxyacyl-COA dehydrogenase; VLCAD = very long-chain acyl-COA dehydrogenase

of CDSP in the mother. ${ }^{7}$ While these women may be asymptomatic on diagnosis, they may be at risk for decompensation if not treated during times of stress.

\section{Carnitine palmitoyltransferase 1A deficiency}

Carnitine palmitoyltransferase 1A (CPT1A) encodes for the homonymous enzyme that resides on the outer mitochondrial membrane. ${ }^{8}$ Its function is to convert long-chain acyl-CoAs to long-chain acylcarnitines, which can be transported into the mitochondria for beta oxidation. Individuals with CPT1A deficiency may present with episodes of hypoketotic hypoglycaemia, acute liver failure, hepatic encephalopathy and hyperammonaemia. It differs from the other LC-FAODs in that cardiac manifestations are very rare in CPT1A deficiency.

\section{Carnitine acylcarnitine translocase deficiency}

Carnitine acylcarnitine translocase is a transporter in the inner mitochondrial membrane to traffic acylcarnitines into the inner mitochondria and free carnitine back towards cytosol. This condition is very rare, with most patients presenting in the neonatal period. Clinical presentation includes hypoketotic hypoglycaemia, hepatomegaly, hyperammonaemia, myotonia, seizures, cardiomyopathy and respiratory distress. ${ }^{1}$ Mortality is high in the neonatal period. Survivors typically experience recurrent rhabdomyolysis. ${ }^{1}$

\section{Carnitine palmitoyltransferase 2 deficiency}

The carnitine palmitoyltransferase 2 gene (CPT2) encodes for an inner mitochondrial membrane protein that cleaves fatty acids from carnitine to be used in beta oxidation. There are three classical presentations of this disorder. The first is a lethal neonatal form that commonly presents shortly after birth with hypoketotic hypoglycaemia, hepatic dysfunction, cardiomyopathy, arrhythmias, encephalopathy and seizures. Structural abnormalities are also seen with dysmorphic facial features, cystic renal dysplasia and neuronal migration defects. ${ }^{9}$ Survival is rare. The second form is an infantile form with recurrent episodes of hypoglycaemia, liver dysfunction, rhabdomyolysis and cardiomyopathy, requiring close monitoring and aggressive treatment for life. The third disorder is characterized primarily by muscle involvement with exercise intolerance and recurrent episodes of rhabdomyolysis. There is also hypotonia and weakness during attacks. ${ }^{10}$ However, many patients are asymptomatic between attacks and some may not present until adulthood.

\section{Very long-chain acyl-CoA dehydrogenase deficiency} very long-chain acyl-COA dehydrogenase (VLCAD) is the most common LC-FAOD. It is due to biallelic variants in ACADVL, which encodes an enzyme involved in the metabolism of acylcarnitines with 14-20 carbons.' Severely affected patients present in the neonatal period with the typical hypoketotic hypoglycaemia, hypotonia, myopathy, hepatic dysfunction, cardiomyopathy and cardiac arrythmias. In the long-term, patients typically experience recurrent rhabdomyolysis and may exhibit cardiac disease progression with significant stress. With the expansion of newborn screening, many more individuals with a milder phenotype are being identified, with symptoms of skeletal myopathy and rhabdomyolysis not presenting until adolescence or adulthood; some may remain asymptomatic for life. ${ }^{11}$ This milder form has now become the most commonly diagnosed phenotype.11

\section{Long-chain 3-hydroxyacyl-CoA dehydrogenase deficiency/trifunctional protein deficiency}

Long-chain 3-hydroxyacyl-COA dehydrogenase (LCHAD) and trifunctional protein (TFP) are two disorders that are clinically indistinguishable. Molecularly, they can be differentiated through the evaluation of HADHA and $H A D H B .{ }^{\prime}$ Most affected patients present in infancy or early childhood with hypoglycaemia, hepatic dysfunction, cardiomyopathy and sudden death. LCHAD may also present with maternal HELLP (haemolysis, elevated liver enzymes, and a low platelet count) syndrome or acute fatty liver of pregnancy when the foetus is affected. Long-term sequelae include myopathy, recurrent rhabdomyolysis and exercise intolerance. Unique phenotypic features of LCHAD/TFP include peripheral neuropathy and retinopathy. ${ }^{12}$ Concern has also been raised for the increased comorbidities of intellectual disability and autism spectrum disorders in LCHAD. ${ }^{13}$ Further research on the underlying mechanism is still required.

\section{Diagnosis}

\section{Biochemical investigations}

General laboratory findings that may be suggestive of a diagnosis of a LC-FAOD include hypoglycaemia accompanied by a lack of, or decrease in, ketone production; an elevation in transaminases; or elevations in creatine kinase. If a medical provider is suspicious of a LC-FAOD, biochemical testing is the best initial step. Diagnostic laboratory studies include plasma total and free carnitine, acylcarnitine profile, urine organic acids and, in some cases, urine acylglycines depending on availability. Table 1 shows the typical biochemical profile for each LC-FAOD. ${ }^{14}$

It is important to consider that a patient, especially one with a mild defect, could have a normal biochemical profile in a well-fed state. This is why the most accurate acylcarnitine profiles for diagnosis are obtained with critical sample laboratory tests during a period of hypoglycaemia. It should also be noted that, if there is a severe defect causing significantly low levels of all carnitine species, the specific diagnostic abnormality may not be evident until the carnitine has been replenished. The wide use of next-generation sequencing has made molecular testing relatively inexpensive, enabling gene-based testing when there is high clinical suspicion. 


\section{Newborn screening}

Newborn screening is now broadly implemented across all states in the USA and in some other countries for LC-FAOD, allowing many patients to be diagnosed before initial presentation. ${ }^{6}$ Newborn screening specimens are typically obtained at 24-48 hours of age when newborns are still in a relatively stressed state from labour and delivery and may not yet be feeding significant amounts. By the time of follow-up testing, they are typically feeding regularly and acylcarnitine abnormalities seen at screening may no longer be evident. Therefore, the acylcarnitine profile alone cannot be used to rule out the diagnosis of LC-FAOD and must be combined with either molecular or enzymatic testing. Early diagnosis, while improving the outcome, does not prevent risk of further decompensations. Furthermore, a normal newborn screen does not completely rule out the possibility of an LC-FAOD, so any patient who presents clinically with suggestive findings should have diagnostic testing.

Newborn screening programmes are giving us a broadened view of phenotype and a more accurate understanding of the incidence of these disorders. The current incidence estimate for all FAODs is 0.9-15.2 per 100,000 , but there may be significant geographic variability. ${ }^{15}$ The newborn screening programme in Tianjin, China, screened 220,443 infants from May 2013 to December of 2018, and diagnosed 15 individuals with FAODs, with the most common being CDSP. ${ }^{16} \mathrm{~A}$ group from Italy also recently published their 5-year screening data, from February 2014 to April 2019. They quote a new regional incidence of 1:4,316 newborns with FAODs, with 20 confirmed diagnoses. ${ }^{17}$ Continued global investigations will further our understanding of the overall prevalence of these disorders.

\section{Acute management}

Patients with LC-FAODs suffer from recurrent acute metabolic decompensations that require emergent medical intervention. Medical providers should counsel patients on the avoidance of triggers of decompensation, such as prolonged fasting, excess activity and emotional stress. However, other stressors, such as intercurrent illnesses or surgery, may not be preventable. ${ }^{4}$ The severity of the disorder will often correlate with the timing of initial decompensation, with the most severe disorders presenting with significant cardiac or hepatic symptoms shortly after birth. During times of acute illness, patients should shorten fasting times and increase carbohydrate caloric intake with frequent glucose-containing beverages. If patients have severe symptoms, poor oral intake or vomiting, it is recommended that they seek immediate medical attention and be started on dextrose-containing intravenous fluids. Ten percent dextrose solution (D10) at 1.5 times maintenance is usually sufficient to stop catabolism; however, this should be titrated with the help of a metabolic provider. ${ }^{18}$ Laboratory studies typically include liver function testing and creatine kinase to assess response to therapy. Additional medical interventions will be illness or stressor specific. Once the patient is in their normal state of health, dextrose fluids should be weaned gradually to prevent a rebound catabolic state from the withdrawal of calories.

\section{Chronic management}

\section{Dietary management}

Management of LC-FAODs typically consists of avoidance of prolonged periods of fasting, dietary restriction of long-chain fats, supplementation with medium-chain triglycerides (MCTs), and a moderate increase in carbohydrate intake. Small snacks may be appropriate prior to any activity or to provide increased calories at times of physical exercise, or at bedtime prior to a longer fasting period. As per the Genetic Metabolic Dieticians International guidelines, it is typically recommended that dietary fat be limited to $20-30 \%$ of total energy intake. ${ }^{19}$ The patient's severity is used to determine how much energy is from long-chain fat versus MCT oil. For example, patients with mild to moderate disease may have their calories split 50/50, while those with a severe LC-FAOD would have a ratio of 2:1 MCTs versus long-chain fat.

The European guidelines allow slightly more liberalization of fats in asymptomatic individuals, with fats constituting $30-40 \%$ of total energy and only 10-15\% from MCTs. ${ }^{15}$ MCTs work well for LC-FAOD treatment, because these shorter-chain fats bypass the metabolic defect, are able to diffuse across the mitochondrial membrane without the requirement of transporters, and then undergo fewer rounds of beta oxidation to feed into the tricarboxylic acid cycle for energy production. ${ }^{15}$ Essential fatty acids should also be monitored and appropriately supplemented. Current dietary therapy guidelines are largely consensus driven. Carnitine supplementation is controversial. Docosahexanoic acid is recommended for LCHAD/TFP, and new research suggests consideration of supplementation in other LC-FAODs as well. ${ }^{20}$ Despite this long-standing therapeutic guidance, even patients with well-controlled symptoms experience recurrent hospitalizations and high morbidity/mortality rates. ${ }^{21}$

\section{Triheptanoin}

Triheptanoin is a highly purified, 7-carbon chain triglyceride that has recently been approved by the US Food and Drug Administration (FDA) for the treatment of LC-FAODs in the USA. The metabolism of odd-chain carbon species allows for broad replenishment of tricarboxylic acid cycle intermediates by supplying both acetyl CoA and propionyl COA, differing from C8 oils that supply acetyl CoA only. In clinical studies, the most common side effects were gastrointestinal: diarrhoea, abdominal pain/discomfort and vomiting, which are the same side effects seen with standard MCTs. ${ }^{22}$ The use of triheptanoin differs slightly from typical US dietary management using MCTs. While traditionally, C8 MCT oil was dosed at $15-25 \%$ of daily caloric intake, the recommended dose range of triheptanoin is $25-35 \%$ of daily caloric intake. ${ }^{23}$

Clinical trials have demonstrated that triheptanoin reduces the incidence of major clinical events (defined as hypoglycaemia, cardiomyopathy or rhabdomyolysis) and hospitalizations in patients with LC-FAODS. ${ }^{20}$ In an independent, double-blind, controlled study, Gillingham et al. randomized 32 patients with LC-FAOD to receive either triheptanoin or C8 oil, both of which being $20 \%$ of the subjects' daily caloric intake. ${ }^{24}$ They found that the triheptanoin group had a $7.4 \%$ increase in left ventricular injection fraction, and a $20 \%$ decrease in left ventricular wall mass. Patients also experienced a lower heart rate for the same amount of work when compared to their $\mathrm{C} 8$ counterparts.

\section{Macronutrients}

While the implementation of triheptanoin has been a major advancement in the management of patients with LC-FAODs, there has also been new research regarding the macronutrient composition of dietary management. The diet traditionally consists of an increase in carbohydrate intake to maintain caloric needs and prevent catabolism of fatty acids. Gillingham et al. recently conducted a 4-month trial in which they compared two groups: one with increased dietary carbohydrate intake and the other with increased dietary protein. Both groups had roughly $20 \%$ of daily caloric intake from fat. ${ }^{25}$ The high carbohydrate group were prescribed daily caloric intake of $11-13 \%$ protein and $64-74 \%$ carbohydrate, while the high protein group were prescribed daily caloric intake of $25-28 \%$ protein and $50-56 \%$ carbohydrate. Although the study was small in size $(n=13)$, they found that the high-protein 
group had increased blood levels of short-chain acylcarnitines, reduced intrahepatic lipid content, and maintained lean body mass while the high-carbohydrate group lost lean body mass.

\section{Investigational therapies}

There are a number of novel approaches for the treatment of LC-FAOD being investigated in clinical or preclinical studies. Reneo Pharmaceuticals is currently recruiting for its phase $\mathrm{lb}$ study on a peroxisome proliferator-activated receptor delta agonist, which, in cell culture systems, increased fatty-acid oxidation and mitochondrial energy metabolism (updates on their study are available on ClinicalTrials.gov). ${ }^{26}$ Work by Bleeker et al. demonstrated improvement in patients with VLCAD with the supplementation of nutritional ketones as an alternative fuel source prior to exercise. ${ }^{27}$ Advancements continue in the realm of gene therapy for common LC-FAODs. ${ }^{28}$

\section{Conclusion}

Recent years have seen significant improvements in our ability to diagnose and manage LC-FAODs. Both implementation of newborn screening and expansion of next-generation sequencing has allowed us to broaden our understanding of the known phenotypes. While these conditions are relatively rare, collectively they are one of the most common metabolic defects. It is critical for all healthcare providers to include these in their differential diagnoses, and not miss a highly treatable condition. ${ }^{1,3}$ As LC-FAODs are increasingly diagnosed, further advances in treatment and management are important to reduce the morbidity and mortality associated with these disorders. Triheptanoin has provided a significant new treatment option in the USA. Further research and novel therapies will likely improve disease management and quality of life for individuals affected by LC-FAODs. ]
1. Vockley J, Longo N, Andresen B, Bennet M. Mitochondrial Fatty Acid Oxidation Defects. In: Sarafoglou K, Hoffmann G, Roth K
(eds.). Pediatric Endocrinology and Inborn Errors of Metaboll

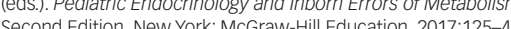

El-Gharbawy A, Vockley J. Inborn errors of metabolism with El-Gharbawy A, Nock rey. Mborn errors of metabolism with myopathy: defects of fatty acid oxidation and the carnitine

3. Nyhhan WL, Barshop BA, Ozand PT. Introduction to Disorders Nyhan WL, Barshop BA, Ozand PT. Introduction to Disorders
of Fatty Acid Oxidation. In: Nyhan WL, Barshop BA, Ozand PT of Fatty Acid Oxidation. In: Nyhan WL, Barshop BA, Ozand PT (eds.). Atlas of
2005:241-5.

4. Spiekerkoetter U, Lindner M, Santer R, et al. Management and outcome in 75 individuals with long-chain fatty acid oxidation defects: results from a workshop. I Inherit Metab Dis. 2009;32:488-97.

5. Li FY, El-Hattab AW, Bawle EV, et al. Molecular spectrum of SLC22A5 (OCTN2) gene mutations detected in 143 subjects evaluated for systemic carnitine deficiency. Hum Mutat 2010:31:E1632-51

6. Wilcken B. Fatty acid oxidation disorders: outcome and long-term prognosis. J Inherit Metab Dis. 2010:33:501-6. long-term prognosis. J Inherit Metab Dis. 2010;33:501-6.
El-Hattab AW, Li FY, Shen J, et al. Maternal systemic primary carnitine deficiency uncovered by newborn screening: clinical, biochemical, and molecular aspects. Genet Med. 2010;12:19-24

8. Knottnerus SJG, Bleeker JC, Wüst RCl, et al. Disorders of mitochondrial long-chain fatty acid oxidation and the carnitine shuttle. Rev Endocr Metab Disord. 2018;19:93-106

9. Boemer F, Deberg M, Schoos R, et al. Diagnostic pitfall in antenatal manifestations of CPT II deficiency. Clin Genet. 2016;89:193-7.

10. Bonnefont JP, Djouadi F, Prip-Buus C, et al. Carnitine palmitoyltransferases 1 and 2: biochemical, molecular and medical aspects. Mol Aspects Med. 2004:25:495-520.
11. Pena LD, van Calcar SC, Hansen J, et al. Outcomes and genotype-phenotype correlations in 52 individuals with VICAD deficiency diagnosed by NBS and enrolled in the IBEM-IS database. Mol Genet Metab. 2016;118:272-81.

12. Fletcher $\mathrm{AL}$, Pennesi ME, Harding $\mathrm{CO}$, et al. Observations regarding retinopathy in mitochondrial trifunctional protein regarding retinopathy in mitochondrial trifunction

13. Strandqvist $A$, Haglind $C B$, Zetterström RH, et al. Neuropsychological development in patients with long-chain 3-hydroxyacyl-COA dehydrogenase (LCHAD) deficiency. JIMD Rep. 2016;28:75-84.

4. Merritt JL 2nd, Norris $M$, Kanungo $S$. Fatty acid oxidation disorders. Ann Trans/ Med. 2018;6:473.

15. Marsden $\mathrm{D}$, Bedrosian CL, Vockley J. Impact of newborn screening on the reported incidence and clinical outcomes associated with medium- and long-chain fatty acid oxidation disorders. Genet Med. 2021;23:816-29.

16. Wang S, Leng J, Diao C, et al. Genetic characteristics and follow-up of patients with fatty acid $\beta$-oxidation disorders through expanded newborn screening in a Northern Chinese

population. J Pedautr Endocrinol Metab. 2020,33.683-90.

characterization and clinical follow up of mitochondrial fatty
chen characterization and clinical follow up oxidation disorders in the new era of expanded newborn acid oxidation disorders in the new era of expanded newborn
screening: a single centre experience. Mol Genet Metab Rep.

8. Aldubayan SH, Rodan LH, Berry GT, Levy HL. Acute illness protocol for fatty acid oxidation and carnitine disorders. Pediatr Emerg Care. 2017;33:296-301

19. Rohr F. Nutrition Management of Fatty Acid Oxidation Disorders. In: Bernstein L, Rohr F, Helm J (eds.). Nutrition Management of Inherited Metabolic Diseases. New York: Springer, 2015:271-82

20. Gillingham MB, Weleber RG, Neuringer M, et al. Effect of optimal dietary therapy upon visual function in children with long-chain 3-hydroxyacyl COA dehydrogenase and trifunctional protein deficiency. Mol Genet Metab. 2005;86:124-33.

21. Vockley J. Long-chain fatty acid oxidation disorders and current management strategies. Am I Manag Care. 2020;26(Suppl. 7):S147-54.

22. Vockley J, Burton B, Berry G, et al. Effects of triheptanoin (UX007) in patients with long-chain fatty acid oxidation disorders: results from an open-label, long-term extension study. J Inherit Metab Dis. 2021;44:253-63.

23. Vockley J, Longo $\mathrm{N}$, Madden $\mathrm{M}$, et al. Dietary management and major clinical events in patients with long-chain fatty aci oxidation disorders enrolled in a phase 2 triheptanoin study. Clin Nutr ESPEN. 2021;41:293-8.

24. Gillingham MB, Heitner SB, Martin J, et al. Triheptanoin versus trioctanoin for long-chain fatty acid oxidation disorders: a double blinded, randomized controlled trial. J Inherit Metab Dis. 2017; $40: 831-43$

25. Gillingham $M B$, Elizondo $G$, Behrend $A$, et al. Higher dietary protein intake preserves lean body mass, lowers liver lipid protein intake preserves lean body mass, lowers IIver lipid long-chain fatty acid oxidation disorders. J Inherit Metab Dis. 2019:42:857-69.

26. ClinicalTrials.gov. A Study of the Safety of RENO01 in Patients with Fatty Acid Oxidation Disorders. ClinicalTrials.gov Identifier: NCT03833128. Available at: https://clinicaltrials.gov/ct2/show/ NCT03833128 (accessed 22 April 2021).

27. Bleeker JC, Visser G, Clarke K, et al. Nutritional ketosis improves exercise metabolism in patients with very long-chain acyl-COA dehydrogenase deficiency. J Inherit Metab Dis. 2020;43:787-99.

28. Zieger M, Keeler AM, Flotte TR, et al. AAV9 gene replacement therapy for respiratory insufficiency in very-long chain acyl-COA dehydrogenase deficiency. J Inherit Metab Dis. 2019;42:870-7. 